\title{
Morfometria de mudas de itaúba (Mezilaurus itauba (Meisn.) Taub. ex Mez) produzidas a partir de substratos alternativos
}

\author{
Morphometry of itaúba seedlings (Mezilaurus itauba (Meisn.) Taub. ex Mez) produced from \\ alternative substrates
}

Morfometría de plántulas de itaúba (Mezilaurus itauba (Meisn.) Taub. Ex Mez) producidas a partir de sustratos alternativos

Recebido: 12/08/2021 | Revisado: 18/08/2021 | Aceito: 22/08/2021 | Publicado: 24/08/2021

Ítalo Felipe Nogueira Ribeiro

ORCID: https://orcid.org/0000-0003-0498-6949 Universidade Federal do Acre, Brasil E-mail: italo080@live.com

Cleverson Agueiro de carvalho

ORCID: https://orcid.org/0000-0002-3256-7023 Universidade Federal do Acre, Brasil

E-mail: cleversoncarvalho92@gmail.com

Reginaldo Almeida Andrade

ORCID: https://orcid.org/0000-0001-6547-4977

Universidade Federal do Acre, Brasil

E-mail: Reginaldo.andrade@unir.br

Felippe Coelho de Souza

ORCID: https://orcid.org/0000-0002-8942-1033

Universidade Federal do Acre, Brasil

E-mail: felippeenf@yahoo.com.br

Rychaellen Silva de Brito

ORCID: https://orcid.org/0000-0002-3387-7218

Universidade Federal do Acre, Brasil

E-mail: rychaellenbrito@gmail.com

Davair Lopes Teixeira Junior

ORCID: https://orcid.org/0000-0001-5051-9255

Universidade Federal do Acre, Brasil

E-mail: davair.junior@ifac.edu.br

Matheus Matos do Nascimento

ORCID: https://orcid.org/0000-0003-0230-0042

Universidade Federal do Acre, Brasil

E-mail: matheusxmattos@gmail.com

\begin{abstract}
Resumo
O uso de substratos alternativos pode viabilizar economicamente a produção de mudas florestais em viveiro, sendo também uma alternativa sustentável para a gestão resíduos orgânicos agroflorestais. A espécie Mezilaurus itauba (Meisn.) Taub. ex Mez ocorre na Amazônia Ocidental, sendo uma árvore de grande porte e alto potencial econômico, explorada pelo setor madeireiro. Esse trabalho visou avaliar os efeitos de substratos orgânicos na produção de mudas de itaúba. Usou-se o delineamento inteiramente casualizado, com quatro tratamentos: $\mathrm{COCB}$ - composto orgânico à base de capim braquiária (1:1), SC - substrato comercial - SC, COCC - composto orgânico + casca de castanha (2:1) e COFC - composto orgânico + farinha de castanha (2:1). Realizou-se a análise das mudas de $M$. itauba através das variáveis: comprimento da parte aérea - CPA, diâmetro do coleto - DC, relação entre o comprimento da parte aérea e o diâmetro do coleto - CPA/DC, massa seca radicular - MSR, da parte aérea - MSPA e total - MST e índice de Dickson - IQD. As mudas produzidas no substrato COCB (1:1) apresentaram maior altura, diâmetro do coleto e acúmulo de massa seca radicular, da parte aérea e total, resultando em um maior valor de índice de Dickson. Observou-se maior robustez nas mudas produzidas no substrato COFC. O substrato comercial originou mudas com menor altura, diâmetro e menor acúmulo de massa seca radicular, da parte aérea e total. $\mathrm{O}$ uso de substratos alternativos, como o composto orgânico, casca e farinha de castanha, favorece o crescimento morfométrico e o acúmulo de biomassa em mudas de $M$. itauba.
\end{abstract}

Palavras-chave: Espécie madeireira; Produção de mudas; Resíduos agroflorestais. 


\begin{abstract}
The use of alternative substrates can make economically viable the production of forest seedlings in nurseries, being also a sustainable alternative for agroforestry organic waste management. The species Mezilaurus itauba (Meisn.) Taub. ex Mez occurs in the Western Amazon, being a large tree with high economic potential, exploited by the timber sector. This work aimed to evaluate the effects of organic substrates on the production of itaúba seedlings. A completely randomized design was used, with four treatments: COCB - organic compost based on signal grass (1:1), SC - commercial substrate - SC, COCC - organic compost + chestnut husk $(2: 1)$ and COFC - organic compost + chestnut flour (2:1). The analysis of $M$. itauba seedlings was carried out using the following variables: shoot length CPA, stem diameter - DC, ratio between shoot length and stem diameter - CPA/DC, root dry mass - MSR, aerial part MSPA and total - MST and Dickson index - IQD. The seedlings produced in the substrate COCB (1:1) showed greater height, stem diameter and accumulation of root, shoot and total dry mass, resulting in a higher Dickson index value. Greater robustness was observed in seedlings produced in the COFC substrate. The commercial substrate originated seedlings with smaller height, diameter and less accumulation of root, shoot and total dry mass. The use of alternative substrates, such as organic compost, nut husk and flour, favors morphometric growth and biomass accumulation in $M$. itauba seedlings.
\end{abstract}

Keywords: Wood species; Seedling production; Agroforestry waste.

\title{
Resumen
}

El uso de sustratos alternativos puede viabilizar económicamente la producción de plántulas en plantas vivas, siendo también una alternativa sustentable para el manejo de residuos orgánicos provenientes de la agroforestería. La especie Mezilaurus itauba (Meisn.) Taub. ex Mez ocurre en la Amazonía Occidental, siendo un árbol de gran tamaño con alto potencial económico, explotado por el sector maderero. Este trabajo tuvo como objetivo evaluar los efectos de sustratos orgánicos en la producción de plántulas de itaúba. Se utilizó un diseño completamente al azar, con cuatro tratamientos: COCB - compost orgánico a base de pasto señal (1: 1), SC - sustrato comercial - SC, COCC - compost orgánico + cascarilla de castaño (2: 1) y COFC - compost orgánico + harina de castaña (2: 1). El análisis de plántulas de $M$. itauba se realizó utilizando las siguientes variables: longitud de brote - CPA, diámetro de tallo - DC, relación entre longitud de brote y diámetro de tallo - CPA / DC, masa seca de raíz - MSR, parte aérea - MSPA y total - MST, y Índice de Dickson - IQD. Las plántulas producidas en el sustrato COCB (1: 1) mostraron mayor altura, diámetro del tallo y acumulación de massa en la raíz brote y parte aérea, resultando en un mayor valor del índice de Dickson. Se observó mayor robustez en plántulas producidas en el sustrato COFC. El sustrato comercial originó plántulas de menor altura, diámetro y menor acumulación massa em la raíz y parte aérea. El uso de sustratos alternativos, como compost orgánico, cascarilla y harina de castaña, favorece el crecimiento morfométrico y la acumulación de biomasa en las plántulas de $M$. itauba.

Palabras clave: Especies de madera; Producción de plántulas; Residuos agroforestales.

\section{Introdução}

A floresta Amazônica fornece diversos serviços ambientais, dentre os quais, a regulação do clima, sequestro e estoque de carbono, conservação do solo e dos recursos hídricos. Todavia, nas últimas décadas, devido a exploração ilegal de madeira, abertura de novas áreas destinadas a pecuária, além da urbanização desordenada, é notório o avanço da fragmentação florestal na região, ameaçando a propagação de inúmeras espécies florestais nativas (Celentano et al. 2018). Diante disso, diversas políticas públicas, promovidas por governos estaduais e federal, vêm sendo implementadas no intuito de frear o desmatamento e estimular a recomposição florestal em áreas degradadas (Celentano et al. 2018; Silva \& Nunes, 2017).

A itaúba (Mezilaurus itauba Taub. ex Mez) é uma das espécies mais visada nos planos de manejo e também na extração ilegal madeireira. A planta é descrita como arbórea climáxica, produz madeira de elevada resistência mecânica, e pode atingir até $40 \mathrm{~m}$ de altura e $80 \mathrm{~cm}$ de diâmetro. Sua madeira é comumente empregada na construção civil, na confecção de postes, dormentes, estacas, dentre outras peças, o que lhe garante alto potencial econômico para serem cultivadas em sistemas agroflorestais, ou mesmo para serem utilizadas em áreas de recomposição florestal (Lorenzi, 2002; Garcia et al. 2012).

A eficiência de projetos de reflorestamento depende da disponibilidade e qualidade de mudas, que precisam ser produzidas em larga escala e rustificadas para sobreviverem em campo. A produção de mudas em viveiro é alternativa viável para suprir essa demanda, tendo como vantagens a possibilidade do controle eficiente de fatores como a luminosidade, temperatura e disponibilidade hídrica, que podem afetar a qualidade da muda produzida. Além disso, permite a utilização de 
recipientes com volumes adequados e substratos específicos que atendam às necessidades morfofisiológicas da espécie cultivada (Haase \& Davis, 2017).

O conhecimento prévio de propriedades físico-químicas de um substrato é essencial para determinar sua viabilidade na produção de mudas. Isso porque, após o fim da reserva nutritiva presente nos tecidos da semente, o crescimento inicial da planta dependerá, dentre outros fatores, da disponibilidade de água e nutrientes do meio onde é cultivada (Oliveira et al. 2016).

Substratos de qualidade devem ser homogêneo, com boa capacidade de retenção de água e elevada capacidade de troca catiônica. Além disso, devem apresentar baixa densidade, permitir boa aeração e drenagem, ser livre de patógenos, bem como ser de baixo custo (Hartmann et al. 2011). O uso de substratos comerciais é recorrente em viveiros comerciais, entretanto, apresenta como desvantagem o elevado custo, por isso, pode ser substituído por substratos alternativos, preparados a partir de resíduos orgânicos diversos (Soares et al. 2014; Almeida et al. 2020).

Como as exigências hídricas e nutricionais da maioria das espécies florestais nativas ainda é desconhecida, torna-se necessário analisar a qualidade das mudas produzidas em diferentes meios, afim de determinar o substrato mais adequado para a produção de mudas de itaúba. Para isso, as avaliações podem ser realizadas através da mensuração de variáveis morfométricas, como comprimento da parte aérea, diâmetro do coleto e massa seca de mudas (Eloy et al. 2013; Gomes et al. 2002).

Diante da importância da espécie e a necessidade do conhecimento em procedimentos relacionados com a produção de mudas de espécies arbóreas nativas, buscando a utilização de materiais renováveis a baixo custo, este trabalho visou avaliar o efeito de substratos orgânicos sobre a produção de mudas de Mezilaurus itauba.

\section{Metodologia}

O experimento foi realizado no Viveiro da Fundação de Tecnologia do Estado do Acre (FUNTAC), situado em Rio Branco, Acre, entre os meses de fevereiro e outubro de 2020. A presente pesquisa aborda aspectos experimentais qualitativos e foi estruturada em ambiente controlado, de forma que os dados produzidos possam ser analisados e validados por modelos estatísticos, garantindo confiabilidade nos resultados, conforme preconiza Pereira et al. (2018). O clima local apresenta duas estações bem definidas, estiagem (abril - outubro) e o inverno amazônico (novembro - março), precipitação média de 1.947 mm e temperatura média de $24^{\circ} \mathrm{C}$ (Ribeiro et al. 2020).

As sementes de itauba foram coletadas de cinco matrizes localizadas no munícipio de Capixaba - AC $\left(63^{\circ} 75^{\prime} 15^{\prime}\right.$ ” W; $88^{\circ} 19^{\prime} 45.3^{\prime \prime}$ S) em setembro de 2019. O delineamento experimental foi inteiramente casualizado, com quatro tratamentos, sendo estes: COCB - composto orgânico à base de capim braquiária (1:1), COCC - composto orgânico de capim braquiária + casca de castanha-do-brasil (2:1), COFC - composto orgânico de capim braquiária + farinha de casca de castanha-do-brasil (2:1), e o substrato comercial (SC), com 30 repetições por tratamento, totalizando 120 unidades experimentais.

O composto orgânico foi preparado por meio pilhas de compostagem formadas exclusivamente por capim braquiária (Brachiaria decumbens), no Sitio Ecológico Seridó, localizado no munícipio de Rio Branco - AC, na latitude de 953’ S e longitude $67^{\circ} 49^{\prime} \mathrm{W}$. As pilhas foram expostas ao ambiente por cerca 120 dias, quando apresentou mudança de coloração para a cor escura, e temperatura inferior a $35^{\circ} \mathrm{C}$, indicando o fim da fase de fermentação e o início da mineralização da matéria orgânica. Esse mesmo composto foi utilizado como base para a preparo dos substratos combinados com resíduos de casca de castanha-do-brasil.

Os substratos preparados a partir das cascas ou farinha de castanha-do-brasil foram elaborados a partir de resíduos doados pela cooperativa de comercialização central do Acre - COOPERACRE. O material foi separado de acordo com a granulometria, a casca de castanha foi peneirada em malha de $5 \mathrm{~mm}$, já a farinha de castanha em malha de $3 \mathrm{~mm}$. O substrato comercial utilizado foi da marca Vivato Plus, composto à base de casca de pinus, vermiculita, moinha de carvão vegetal, água 
e espuma fenólica.

A caracterização físico-química dos substratos foi realizada pelo laboratório de análise do solo e planta do Instituto Agronômico de Campinas (IAC) (Tabelas 1, 2 e 3). As propriedades físicas foram obtidas mediante procedimentos descritos nas instruções normativas 17 e 31, de 21 de maio de 2007 e 23 de outubro de 2008, do Ministério de Estado da Agricultura, Pecuária e Abastecimento, respectivamente, com exceção da porosidade, que foi estimada pelo método European Standart.

Tabela 1. Propriedades físicas dos substratos à base de composto orgânico de capim braquiária (COCB), farinha de castanhado-brasil (COFC), casca de castanha-do-brasil (COCC) e substrato comercial (SC) utilizados para a produção de mudas de itaúba.

\begin{tabular}{|c|c|c|c|c|c|c|}
\hline \multirow{2}{*}{ Substrato } & $\mathrm{CE}$ & UM & DU & CRA $^{10}$ & CTC & PT \\
\hline & ds $m^{-1}$ & $\% \mathbf{m} / \mathbf{m}$ & $\mathrm{kg} \mathrm{m}^{-3}$ & $\% \mathrm{~mm}$ & mmolc $\mathbf{d m}^{-3}$ & $\% \mathrm{v} / \mathrm{v}$ \\
\hline COCB & 0,5 & 46,4 & 782,3 & 140,5 & 185,8 & 82,2 \\
\hline COFC & 0,8 & 39,3 & 686,9 & 159,7 & 301,7 & 79,6 \\
\hline $\mathrm{COCC}$ & 0,7 & 37,5 & 743,7 & 99,0 & 231,3 & 77,0 \\
\hline SC & 1,3 & 39,6 & 451,9 & 292,0 & 200,0 & 85,4 \\
\hline
\end{tabular}

Onde: $\mathrm{CE}=$ Condutividade elétrica; $\mathrm{UM}=$ Umidade; $\mathrm{DU}=$ Densidade úmida CRA = Capacidade de retenção de água; CTC = Capacidade de troca catiônica e PT = Porosidade Total. Fonte: IAC (2020).

A determinação das concentrações dos macros e micronutrientes (Tabela 2 e Tabela 3) presentes em cada substrato foi feita pelo método de extração nítrico-perclórico, exceto para o nitrogênio, que foi quantificado pelo método de digestão de Kjeldahl, e teor de carbono orgânico foi mensurado pelo método de Walkley-Black.

Tabela 2. Valores de $\mathrm{pH}$ e teor de macronutrientes dos substratos à base de composto orgânico à base de capim braquiária (COCB), farinha de castanha (COFC), casca de castanha (COCC) e substrato comercial (SC) utilizados para produção de mudas de itaúba.

\begin{tabular}{|c|c|c|c|c|c|c|}
\hline \multirow[b]{2}{*}{ Substrato } & \multirow[b]{2}{*}{ pH } & $\mathbf{N}$ & $\mathbf{P}$ & $\mathbf{K}$ & $\mathrm{Ca}$ & Mg \\
\hline & & \multicolumn{5}{|c|}{$\mathrm{g} \mathrm{kg}^{-1}$} \\
\hline COCB & 5,8 & 6,3 & 0,7 & 3,4 & 3,0 & 1,6 \\
\hline $\mathrm{COFC}$ & 5,0 & 8,2 & 0,7 & 5,5 & 2,4 & 1,4 \\
\hline COCC & 5,0 & 5,8 & 0,7 & 5,3 & 2,6 & 1,3 \\
\hline $\mathrm{SC}$ & 5,6 & 4,0 & 2,5 & 6,9 & 10,4 & 14,4 \\
\hline
\end{tabular}

Onde: $\mathrm{pH}$ = Potencial Hidrogeniônico; $\mathrm{N}$ = Nitrogênio; $\mathrm{P}$ = Fosforo; $\mathrm{K}=$ Potássio; Ca = Cálcio; Mg = Magnésio. Fonte: IAC (2020).

Tabela 3. Valores de teor de micronutrientes e relação $\mathrm{C} / \mathrm{N}$ dos substratos à base de composto orgânico à base de capim braquiária (COCB), farinha de castanha (COFC), casca de castanha (COCC) e substrato comercial (SC) utilizados para produção de mudas de itaúba.

\begin{tabular}{|c|c|c|c|c|c|c|}
\hline \multirow{2}{*}{ Substrato } & $\mathbf{F e}$ & Mn & B & $\mathrm{Cu}$ & Zn & $\mathrm{C} / \mathrm{N}$ \\
\hline & \multicolumn{2}{|c|}{$\mathrm{g} \mathrm{kg}^{-1}$} & \multicolumn{3}{|c|}{$\mathrm{mg} \mathrm{kg}^{-1}$} & Relação \\
\hline COCB & 9,7 & 1,0 & 49,6 & 8,6 & 57,4 & $16,9: 1,0$ \\
\hline $\mathrm{COFC}$ & 7,5 & 0,6 & 37,2 & 14,6 & 43,6 & $21,5: 1,0$ \\
\hline $\mathrm{COCC}$ & 7,3 & 0,7 & 39,1 & 9,3 & 43,7 & $39,5: 1,0$ \\
\hline $\mathrm{SC}$ & 10,7 & 0,5 & 114,6 & 29,7 & 190,7 & $83,2: 1,0$ \\
\hline
\end{tabular}

Onde: $\mathrm{Fe}=$ Ferro; $\mathrm{Mn}=$ Manganês $; \mathrm{B}=$ Boro $\mathrm{Cu}=$ Cobre; $\mathrm{Zn}=$ Zinco e C/N = Relação carbono e nitrogênio. Fonte: IAC (2020). 
A repicagem das mudas para tubetes de polipropileno atóxico preto de $180 \mathrm{~cm} 3$ ocorreu 30 dias após a semeadura, onde permaneceram durante 120 dias no viveiro coberto com tela monofilamentosa capaz de filtrar $50 \%$ da luminosidade incidente. Durante o período, as mudas foram irrigadas diariamente, com lâmina de $12 \mathrm{~mm}$. Não foram realizadas adubações de plantio ou cobertura. Aos 150 dias após a semeadura as mudas foram submetidas as seguintes análises morfométricas: comprimento da parte aérea (CPA) aferição realizada com régua graduada, diâmetro do coleto (DC) com o auxílio de um paquímetro digital.

Posteriormente as amostras foram inseridas em embalagens de papel Kraft e armazenadas em estufa de ventilação forçada $\left(65^{\circ} \mathrm{C}\right)$, até atingir massa constante. Após secagem, foram determinados a massa seca total (MST), massa seca da parte aérea (MSPA) e massa seca da raiz (MSR) em balança semi-analítica $(0,01 \mathrm{~g})$. Os valores obtidos foram tabulados e calculado o Índice de Dickson et al. (1960), usando a seguinte equação:

$$
\mathrm{IQD}=\frac{\mathrm{MST}(\mathrm{g})}{\left(\frac{\mathrm{CPA}(\mathrm{cm})}{\mathrm{DC}(\mathrm{mm})}+\frac{\mathrm{MSPA}(\mathrm{g})}{\operatorname{MSR}(\mathrm{g})}\right)}
$$

Onde, MST = Massa Seca Total (g); CPA/DC = Relação do Comprimento da Parte Aérea $(\mathrm{cm})$ sobre o Diâmetro a Altura do Coleto $(\mathrm{mm})$ e MSPA/MSR = Relação da Massa Seca da Parte Aérea (g) sobre a Massa seca da Raiz (g).

$\mathrm{O}$ conjunto de dados foram submetidos à verificação da presença de outliers pelo teste de Grubbs (1969), à normalidade dos resíduos pelo teste de Shapiro e Wilk (1965) e homogeneidade das variâncias pelo teste de Cochran (1947). Posteriormente, as médias de cada tratamento foram submetidas a análise de variância pelo teste $\mathrm{F}$ e comparação pelo teste de Tukey (1953) a 5\% de probabilidade.

\section{Resultados e Discussão}

As características morfométricas das mudas de itaúba foram significativamente afetados $(\mathrm{p}<0,05)$ pela composição dos substratos, com coeficientes de variação entre as médias inferior a $20 \%$ (Tabela 4), indicando alta confiabilidade no modelo utilizado (Garcia, 1989).

Tabela 4. Resumo da análise de variância para o comprimento da parte aérea (CPA), diâmetro do coleto (DC), relação comprimento da parte aérea sobre diâmetro do coleto (CPA/DC), massa seca da parte aérea (MSPA), massa seca radicular (MSR), massa seca total (MST) e índice de Dickson (IQD) das mudas de itaúba em diferentes substratos aos 150 dias após a semeadura.

\begin{tabular}{ccccccccc}
\hline $\begin{array}{c}\text { Fonte de } \\
\text { variação }\end{array}$ & \multirow{2}{*}{ GL } & CPA $(\mathbf{c m})$ & DC $(\mathbf{m m})$ & CPA/DC & MSPA $(\mathbf{g})$ & MSR $(\mathbf{g})$ & MST $(\mathbf{g})$ & IQD \\
\cline { 3 - 8 } Quadrado médio & & \\
\hline Substrato $(\mathrm{S})$ & 3 & $65,5927^{*}$ & $0,9281^{*}$ & $2,1472^{*}$ & $0,7363^{*}$ & $0,5973^{*}$ & $2,6063^{*}$ & $0,3887^{*}$ \\
Resíduo & 36 & 0,48 & 0,0033 & 0,8525 & 0,0028 & 0,0077 & 0,009 & 0,0003 \\
\hline CV $(\%)$ & & 4.04 & 2.07 & 4.72 & 9.69 & 15.02 & 8.37 & 11.74 \\
\hline Média geral & & 17,15 & 2.77 & 6,18 & 0,55 & 0,58 & 1,13 & 0,16 \\
\hline
\end{tabular}

Onde: $*=$ Significativo a $5 \%$ de probabilidade. Fonte: Autores (2020).

O comprimento médio da parte aérea das mudas de itaúba, aos 120 dias após a germinação foi de $17 \mathrm{~cm}$ (Tabela 4), com variações entre $14,17 \mathrm{~cm}$, quando as mudas foram cultivadas no substrato comercial (SC) a 20,31 cm no composto orgânico de capim braquiária COCB (1:1), ou seja, incremento de 30,23\% (Tabela 5). Os resultados para o diâmetro do coleto foram similares, onde o COCB (1:1) promoveu aumento de $22,8 \%$ em ralação ao tratamento SC. 
Tabela 5. Valores médios de comprimento da parte aérea (CPA), diâmetro do coleto (DC), relação comprimento da parte aérea sobre diâmetro do coleto (CPA/DC), massa seca da parte aérea (MSPA), radicular (MSR) e total (MST), e índice de Dickson (IQD). Composto orgânico à base de capim braquiária (COCB), composto orgânico e farinha de castanha (COCF), composto orgânico e casca de castanha (COCC) e substrato comercial (SC).

\begin{tabular}{lccccccc}
\hline Substratos & $\begin{array}{c}\text { CPA } \\
(\mathbf{c m})\end{array}$ & $\begin{array}{c}\text { DC } \\
(\mathbf{m m})\end{array}$ & CPA/DC & $\begin{array}{c}\text { MSPA } \\
(\mathbf{g})\end{array}$ & $\begin{array}{c}\text { MSR } \\
(\mathbf{g})\end{array}$ & $\begin{array}{c}\text { MST } \\
(\mathbf{g})\end{array}$ & IQD \\
\hline COCB $(1: 1)$ & $20,31 \mathrm{~A}$ & $3,07 \mathrm{~A}$ & $6,61 \mathrm{C}$ & $0,93 \mathrm{~A}$ & $0,94 \mathrm{~A}$ & $1,87 \mathrm{~A}$ & $0,25 \mathrm{~A}$ \\
COFC $(2: 1)$ & $16,42 \mathrm{C}$ & $2,92 \mathrm{~B}$ & $5,63 \mathrm{~A}$ & $0,46 \mathrm{~B}$ & $0,52 \mathrm{~B}$ & $0,98 \mathrm{~B}$ & $0,15 \mathrm{~B}$ \\
COCC $(2: 1)$ & $17,69 \mathrm{~B}$ & $2,72 \mathrm{C}$ & $6,52 \mathrm{C}$ & $0,52 \mathrm{~B}$ & $0,46 \mathrm{~B}$ & $0,98 \mathrm{~B}$ & $0,13 \mathrm{C}$ \\
SC & $14,17 \mathrm{D}$ & $2,37 \mathrm{D}$ & $5,99 \mathrm{~B}$ & $0,29 \mathrm{C}$ & $0,41 \mathrm{C}$ & $0,70 \mathrm{C}$ & $0,10 \mathrm{D}$ \\
\hline CV $(\%)$ & 4,04 & 2,07 & 4,72 & 9,69 & 15,02 & 8,37 & 11,74 \\
\hline
\end{tabular}

Letras diferentes indicam diferença significativa a $5 \%$ de probabilidade na coluna. Fonte: Autores (2020).

O maior crescimento morfométrico das plantas produzidas em composto orgânico de capim braquiária pode ser explicado pela baixa relação C/N do material, 16,9:1,0 (Tabela 3). Segundo Sarma e Gogoi (2015), substratos orgânicos com relação C/N entre 10:1,0 e 20:1,0 favorecem o crescimento vegetal em altura e diâmetro, visto que nessa faixa ocorre maior taxa de mineralização, e consequentemente, maior liberação de nutrientes para as plantas. Por outro lado, Brietzke (2016) mencionam que relações superiores a 50, como visto no substrato comercial, promovem imobilização de $\mathrm{N}$ mediado por microorganismos, podendo alterar alguns parâmetros fisiológicos da planta, como redução da fotossíntese e da translocação de fotoassimilados entre o eixo aéreo e radicular (Mendes et al. 2019), inibindo o crescimento em altura e diâmetro. Substratos com elevadas relações $\mathrm{C} / \mathrm{N}$ também possuem baixa taxa de mineralização, reduzindo a disponibilidade de nutrientes em forma assimilável às plantas a curto prazo, tornando o crescimento inicial mais lento (Sarma \& Gogoi, 2015).

Além da alta relação $\mathrm{C} / \mathrm{N}$, o elevado teor de Boro também pode ter limitado o crescimento em altura e diâmetro do coleto nas mudas produzidas em substrato comercial (Tabela 3). O Boro é um micronutriente essencial para as plantas, atuando no transporte de açúcares, composição da parede celular e atua no processo de floração, entretanto quando em excesso, promove a formação de radicais de superóxido, danificando a membrana celular (Kirkby e Römheld, 2007). Araújo et al. (2017), relatam que o mogno africano (Khaya senegalensis) apresenta redução no crescimento em altura da parte aérea e diâmetro quando exposto a doses de B superiores a $2 \mathrm{mg} \mathrm{kg}^{-1}$, e que a demanda por esse micronutriente varia de acordo com a espécie, bem como as doses ótima e de toxidez.

A relação cálcio/magnésio no substrato comercial, 0,7:1,0 (Tabela 2) também pode ter acarretado respostas negativas no crescimento das mudas. Segundo Salvador et al. (2011), o excesso de um destes nutrientes, ou o desequilíbrio entre ambos, pode inibir a absorção do outro, sendo recomendada a proporção Ca:Mg em torno de 3:1, onde valores abaixo disso podem gerar desequilíbrio nutricional, afetando o crescimento das plantas. O cálcio, assim como o magnésio, são elementos bivalentes, e concorrem pelos mesmos sítios de absorção na planta, e apesar de ser um macronutriente secundário, é um importante componente estrutural da planta, estando presente na parede celular, além disso, um dos indicadores de deficiência é a redução do desenvolvimento de raízes e da parte aérea, além de sintomas visuais nas folhas (Taiz et al. 2017).

As mudas produzidas no substrato formulado à base de composto orgânico de capim braquiária e farinha de castanha (2:1), apresentaram a menor relação entre o comprimento da parte aérea e o diâmetro do coleto, indicando maior robustez (Tabela 5). Esta variável é um indicador de equilíbrio no desenvolvimento das partes da planta, visto se tratar da relação entre dois parâmetros morfológicos importantes, o comprimento da parte aérea, que é referencial de crescimento inicial, e o diâmetro do coleto, que indica a probabilidade de a muda sobreviver em campo, sendo desejado que essa relação seja o menor possível (Cadorin et al. 2015). Segundo Almeida et al. (2020), as relações CPA/DC devem estar entre 5,4 e 8,1, sendo que este valor pode variar de acordo com a espécie, entretanto, não deve ser superior a 10, o que seria um indicativo de estiolamento, 
elevando os riscos de tombamento após o transplante para o campo.

Desta forma, a maior robustez observada nos substratos formulados à base de composto orgânico e farinha de castanha é um indicativo de equilíbrio na translocação de assimilados e acúmulo de massa entre o eixo radicular e aéreo, sendo que a zona de transição entre essas duas partes, denominada coleto, constitui-se um reservatório temporário de assimilados (Afonso et al. 2017).

As plantas cultivadas no composto orgânico de capim braquiária (1:1) propiciou a maior produção de massa radicular, sendo o valor observado nesse tratamento 56,3\% superior ao do substrato comercial (Tabela 5). Com o aumento do desenvolvimento da parte aérea, a planta adota uma estratégia de expandir seu sistema radicular para suportar a parte aérea e ocupar um volume maior no tubete, absorvendo mais nutrientes e água, fato já foi observado por Ferreira et al. (2017), em estudo com a itaúba. Além disso, como já mencionado, a relação Ca:Mg mais equilibrada no substrato alternativo pode ter favorecido o desenvolvimento, não só a parte aérea, mas também o sistema radicular.

O substrato comercial apresentou o menor $(\mathrm{p}<0,05)$ valor de massas secas radicular $(0,41 \mathrm{~g})$, aérea $(0,29 \mathrm{~g})$ e total $(0,70 \mathrm{~g})$. Nas mudas produzidas nesse substrato observa-se maior acúmulo de massa seca na raiz em comparação com a parte aérea, podendo ser uma estratégia da espécie para superar o déficit de N, causado pela alta relação C/N (Brietzke, 2016), realocando mais carbono no eixo radicular, afim de absorver nutrientes nas camadas inferiores do recipiente, o que acarreta na redução de massa seca da parte aérea (Rosado, 2017). A alocação de massa seca vegetal deve ocorrer de forma proporcional entre os órgãos da planta, afim de conferir maior resistência a condições ambientais adversas após o transplantio (Afonso et al. 2017).

Plantas produzidas em composto orgânico de capim braquiária (1:1) apresentaram valores de massa seca total 2,7 vezes maior que aquelas produzidas no substrato comercial (Tabela 5). Os resultados obtidos neste estudo corroboram com os de Almeida et al. (2020), que constataram que o uso de compostos orgânicos na formulação de substratos beneficia o acú mulo de carbono na parte aérea das plantas.

As mudas produzidas em composto orgânico (1:1) registraram, em média, qualidade $60 \%$ superior às mudas produzidas substrato comercial (Tabela 5). Dentre todas variáveis de qualidade de mudas, o índice de Dickson pode ser considerado um dos mais precisos e assertivos, por englobar variáveis alométricas, como a robustez e equilíbrio da distribuição da massa na planta (Eloy et al. 2013; Reis et al. 2016).

Destaca-se que as mudas de itaúba produzidas em substrato com casca ou farinha de castanha-do-brasil apresentaram qualidade superior às produzidas em substrato comercial (Tabela 5), sendo válido ressaltar que a casca e farinha de castanha liberam nutrientes lentamente, sendo uma alternativa para suprir a necessidade da espécie a longo prazo (Nascimento, 2012).

Santos et al. (2018), sugerem a redução da granulometria da casca de castanha como forma de melhorar propriedades físico-químicas deste substrato. É importante salientar que o tamanho das partículas é um fator importante no processo de decomposição. A redução do tamanho dessas partículas aumenta sua área superficial especifica e potencializa a decomposição, acelerando a liberação de nutrientes. Soares et al. (2014), por sua vez, informam que a casca de castanha possui altos teores de $\mathrm{N}$ e K, macronutrientes essenciais para o desenvolvimento vegetal. Sendo assim, acredita-se reduzir sua granulometria e combinar a casca de castanha com materiais de menor densidade, além de ser uma alternativa para a reutilização deste resíduo, pode resultar na confecção de substratos com propriedades físico-químicas apropriadas (Oliveira, 2017).

De acordo com Pascual et al. (2018), as limitações de uso de resíduos orgânicos podem ser resolvidas quando previamente conhecidas, sendo que, de maneira geral a adição de compostos orgânicos em substratos tende a melhorar condições físicas e químicas do meio, beneficiando o crescimento da planta. Entretanto, é necessário padronizar e esterilizar a forma de produção do substrato orgânico, afim de obter um material homogêneo e isento de patógenos.

O uso de substratos alternativos na produção de mudas em viveiros possui enorme potencial, entretanto, existe uma 
lacuna comunicativa entre os produtores de resíduos orgânicos e a demanda de viveiros, causada pela falta de comunicação, que será sanada somente através de pesquisas científicas e troca de informações (Pascual et al. 2018).

\section{Conclusão}

Nas condições edafoclimáticas do estado do Acre, o desenvolvimento inicial de mudas de itaúba é favorecido com uso de substratos alternativos a base de resíduos orgânicos de capim braquiária, casca e farinha de castanha-do-brasil.

O substrato preparado à base de composto orgânico de capim braquiária (1:1) promove mudas de itaúba com maior altura, diâmetro e acúmulo de biomassa, sendo uma alternativa de baixo custo ao substrato comercial.

A casca ou farinha de castanha-do-brasil, adicionado ao composto orgânico de capim braquiária apresentam elevado potencial de uso como substrato, visto que as mudas de itaúba produzidas com estes materiais apresentam melhores indicadores morfométricos e Índice de Qualidade de Dickson que o substrato comercial.

Apesar dos resultados promissores, novos estudos, avaliando os efeitos dos substratos em outras espécies florestais, e também sua influência na sobrevivência e desenvolvimento das mudas à campo precisam ser realizadas para garantir conclusões mais precisas.

\section{Referências}

Almeida, D. M., Silva, B. R. F., Ucella Filho, J. G. M., Sousa, N. A. \& Costa, T. L. N. (2020). Efeito de diferentes substratos no desenvolvimento inicial de mudas de Handroanthus impetiginosus (Mart. ex DC). Brazilian Journal of Development, 6 (5), 24619-24631.

Araújo, M. S., Melo, M. A., Hodecker, B. E. R., Barretto, V. C. M. \& Rocha, E. C. (2017). Adubação com boro no crescimento de mudas de mogno-africano. Revista de Agricultura Neotropical, 4 (1), 1-7.

Brietzke, D. T. (2016). Avaliação do processo de compostagem considerando a relação carbono/nitrogênio. Trabalho de Conclusão de Curso, Universidade do Vale do Taquari, Lajeado, Rio Grande do Sul, Brasil.

Cadorin, D. A., Malavasi, U. C., Coutinho, P. W. R., Dranski, J. A. L. \& Malavasi, M. M. (2015). Metil jasmonato e flexões caulinares na rustificação e crescimento inicial de mudas de Cordia trichotoma. Cerne, 21 (4), 657-664.

Celentano, D., Miranda, M. V. C., Mendonça, E. N., Rousseau, G. X., Muniz, F. H., Loch, V. C., Varga, I. V. D., Freitas, L.; Araújo, P., Narvaes, I. S., Adami, M., Gomes, A. R., Rodrigues, J. C., Kahwage, C., Pinheiro, M. \& Martins, M. B. (2018). Desmatamento, degradação e violência no "Mosaico Gurupi" - A região mais ameaçada da Amazônia. Estudos avançados, 32 (92), 315-339.

Cochran, W. G. (1947). The distribution of the largest of a set of estimated variances as a fraction of their total. Annals of Eugenics, 22 (11), 47-52.

Dickson, A., Leaf, A. L. \& Hosner, J. F. (1960). Quality appraisal of white spruce and white pine seedling stock in nurseries. The Forestry Chronicle, 36 (1), $10-13$.

Eloy, E., Caron, B. O., Schmidt, D., Behling, A., Schwers, L. \& Elli, E. F. (2013). Avaliação da qualidade de mudas de Eucalyptus grandis utilizando parâmetros morfológicos. Floresta, 43 (3), 373-384.

Ferreira, M. S., Santos, J. Z. L., Tucci, C. A. F. \& Costa, L. V. (2017). Crescimento inicial de itaúba e macacaúba em recipientes de diferentes tamanhos. Ciência Florestal, 27 (2), 499-508.

Garcia, C. H. (1989). Tabelas para classificação do coeficiente de variação. IPEF. 12 p. (Circular Técnica, 171).

Garcia, F. M., Manfio, D. R., Sansígolo, C. A. \& Magalhães, P. A. D. (2012). Rendimento no Desdobro de Toras de Itaúba (Mezilaurus itauba) e Tauari (Couratari guianensis) Segundo a Classificação da Qualidade da Tora. Floresta e Ambiente, 19 (4), $468-474$.

Gomes, J. M., Couto, L., Leite, H. G., Xavier, A. \& Garcia, S. L. R. (2002). Parâmetros morfológicos na avaliação de qualidade de mudas de Eucalyptus grandis. Revista Árvore, 26 (6), 655-664.

Grubbs, F. (1969). Procedures for detecting outlying observations in samples. Technometrics, 11 (1), 1-21.

Haase, D. L. \& Davis, A. S. (2017). Developing and supporting quality nursery facilities and staff are necessary to meet global forest and landscape restoration needs. Reforesta, 4 (1), 69-93.

Hartmann, H. T. \& Kester, D. E. (2011). Plant propagation: principles and practices. (8a ed.). Prentice Hall. 915 p.

Kirkby, E. A. \& Römheld, V. (2007). Micronutrientes na fisiologia de plantas: Funções, absorção e mobilidade. Piracicaba: International Plant Nutrition Institute, 24 p. (Encarte Técnico, 118).

Lorenzi, H. (2002). Árvores brasileiras - Manual de identificação e cultivo de plantas arbóreas nativas do Brasil. Ed. Plantarum. 368 p. 
Mendes, R. F., Araújo, J. C., Andrade Neto, R. C., Araújo, J. M. \& Guilherme, J. P. M. (2019). Crescimento de mudas de maracujazeiro em substrato alternativo com fertilizante de liberação controlada. Revista Brasileira de Agropecuária Sustentável, 9 (4), 34-40.

Nascimento, V. F. (2012). Caracterização de biomassas amazônicas - Ouriço de castanha-do-brasil, ouriço de sapucaia e caroço do fruto do tucumã Visando sua utilização em processos de termoconversão. Dissertação de mestrado, Universidade Estadual de Campinas, Campinas, São Paulo, Brasil.

Oliveira, M. C., Ogata, R. S., Andrade, G. A., Santos, D. S., Souza, R. M., Guimarães, T. G., Silva Júnior, M. C., Pereira, D. J. S. \& Ribeiro, J. F. (2016). Manual de viveiro e produção de mudas: espécies arbóreas nativas do Cerrado. Brasília: Embrapa. 124 p.

Pereira, A. S., Shitsuka, D. M., Parreira, F. J., \& Shitsuka, R. (2018). Metodologia da Pesquisa Cientifica. UFSM.

Pascual, J. A., Ceglie, F., Tuzel, Y., Koller, M., Koren, A., Hitchings, R. \& Tittarelli, F. (2018). Organic substrate for transplant production in organic nurseries. A review. Agronomy for Sustainable Development, 38 (3), 1-23.

Reis, S. M., Marimon-Júnior, B. H., Morandi, P. S., Santos, C. O., Oliveira, B. \& Marimon, B. S. (2016). Desenvolvimento inicial e qualidade de mudas de Copaifera langsdorffii Desf. sob diferentes níveis de sombreamento. Ciência Florestal, 26 (1), 11-20.

Ribeiro, Í. F. N., Lima, B. G. \& Ferreira, E. J. L. (2020). Características florísticas e densidade do banco de sementes do solo de uma floresta aberta com bambu no leste do Acre. Scientia Naturalis, 2 (2), 842-850.

Rosado, S. I. P. (2017). Crescimento, estado nutricional e fotossíntese de Cedrela odorata l. adubada com diferentes proporções de nitrato e amônio. Dissertação de mestrado, Universidade Federal do Amazonas, Manaus, Amazonas, Brasil.

Salvador, J. T., Carvalho, T. C. \& Lucchesi, L. A. C. (2011). Relações cálcio e magnésio presentes no solo e teores foliares de macronutrientes. Revista Acadêmica Ciências Agrárias e Ambiental, 9 (1), 27-32.

Santos, J. P., Braga, L. F., Ruedell, C. M., Seben Júnior, G. F., Ferbonink, G. F. \& Caione, G. (2018). Caracterização física de substratos contendo resíduos de cascas de amêndoas de castanha-do-brasil (Bertholletia excelsa H.B.K.). Revista de Ciências Ambientais, 12 (2), 7-17.

Sarma, B. \& Gogoi, N. (2015). Germination and seedling growth of Okra (Abelmoschus esculentus L.) as influenced by organic amendments. Cogent Food \& Agriculture, 1 (1), 1-6.

Shapiro, S. S. \& Wilk, M. B. (1965). An analysis of variance test for normality (complete samples). Biometrika, 52 (3-4), 591-611.

Silva, D. \& Nunes, S. (2017). Avaliação e modelagem econômica da restauração florestal no Estado do Pará. Imazon. 92 p.

Soares, I. D., Paiva, A. V., Miranda, R. O. V. \& Maranho, A. S. (2014). Propriedades físico-químicas de resíduos agroflorestais amazônicos para uso como substrato. Nativa, 2 (3), 155-161.

Taiz, L., Zeiger, E., Moller, I. \& Murphy, A. (2017). Fisiologia e desenvolvimento vegetal. (6a ed.), Artmed. 888 p.

Tukey, J. W. (1953). The problem of multiple comparisons. New Jersey: Princeton University. 189 p. 